\title{
Semi-Blind Source Separation in a multi-user transmission system with Interference Alignment
}

\author{
Yasser Fadlallah*‡ Abdeldjalil Aïssa-El-Bey*‡ Senior Member, IEEE, Karim Abed-Meraim ${ }^{\dagger}$ Senior \\ Member, IEEE, Karine Amis* ${ }^{*}$ Member, IEEE and Ramesh Pyndiah* ${ }^{* \ddagger}$ Senior Member, IEEE \\ *Institut Télécom; Télécom Bretagne; UMR CNRS 3192 Lab-STIC, Technopôle Brest-Iroise CS 8381829238 Brest, France \\ †Polytech'Orléans/PRISME Lab., Université d'Orléans, 12 rue de Blois, BP 6744, 45067 Orléans, France \\ †Université européenne de Bretagne
}

\begin{abstract}
In this paper we address the decoding problem in the $K$-user MIMO interference channel assuming an interference alignment (IA) design. We aim to decode robustly the desired signal without having a full Channel State Information (CSI) (i.e. precoders knowledge) at the receivers. We show the equivalency between the IA model and the Semi-Blind Source Separation model (SBSS). Then, we prove that this equivalence allows the use of techniques employed in source separation for extracting the desired signal free of interference, even though dependency exists between some components of the source signal in the SBSS model. Our simulation results illustrate a BER performance very close to the MMSE receiver with full-CSI.
\end{abstract}

\section{INTRODUCTION}

Interference Alignment (IA) is an interference management strategy that aims to achieve the Degrees of Freedom (DoF) of the $K$-user interference channel (IC). This technique results in a linearly scaling network sum-rate with the number of users sharing a common transmission medium. Its basic idea is a joint design of all transmitted signals such that interfering signals at each receiver overlap and remain distinct from the desired signal [1], [2]. In a $K$-user $M \times N$ MIMO system, where $M$ and $N$ are the number of the transmit and receive antennas, respectively, the feasibility of the IA design has been studied [3], and distributed IA-achieving algorithms have been derived such as in [5]. These algorithms are based on an iterative processing at the transmitter when the full-CSI is provided, and on information exchange with the receiver when only the local CSI is provided.

In this paper, we address the decoding problem when an IA design is employed at the transmitters. That is, the interfering signals are aligned in a subspace linearly independent of the desired signal subspace. The traditional linear decoders estimate the decoding matrices, defined as the interference null space, using Least Square (LS) method essentially based on a training sequence within each frame. Our work concerning the decoding scheme pursues a different approach. The main contribution consists in separating the desired signal from the interference using higher-order cumulants, and then the desired signal is identified using a few training symbols. This approach has the advantage of allowing to decode the received signal even when the number of training symbols is low, and results in a more robust decoding scheme for a same training sequence length. We first show the equivalence between the MIMO IC model proposed herein and a determined semi
Blind Source Separation (SBSS) model when the IA scheme is employed. Then, we demonstrate the ability of the SBSS problem to separate the desired signal from the interference through a joint diagonalization of the fourth-order cumulant matrices [9]. The separation ability is due to the existing independence between the desired signal and the interference. The joint diagonalization is able to extract the desired streams blindly up to a permutation and scaling ambiguities. These ambiguities are revealed using a few training symbols within each transmitted frame, hence, the term semi-blind comes by.

One proposed application of our framework is the communication between $K$ base stations (BS) and $K$ end-users in the downlink mode assuming an IA design. The transmission is assumed in TDD mode. The channel is estimated at the BS using an $N_{s}$-length uplink reference signal sent from the users assuming a reciprocal channel, that is supposed constant during one frame transmission. This strategy is employed in the TDD-uplink transmission scheme in the 3GPP-LTE network [16]. Another strategy that can be used is when each user estimates the channels connecting him with the BS, and feeds it back quantized to them BS. The reliability of this strategy depends on the channel estimator at the receiver, the channel quantizer, and the feedback link quality. Research works carried out, e.g. in [6], and have studied the IA achievability using a limited feedback link. They have defined a channel quantizer over the composite Grassmannian manifold, that achieves a full DoF in the IC when the feedback bit rate scales sufficiently fast with the SNR. In our study, we assume an IA-achieving design. Our simulation results highlight the robustness of the proposed decoding strategy when both perfect and imperfect IA design hold at the transmitters.

This paper is organized as follows. In Section II, the assumed MIMO IC is described. A BSS model associated to the MIMO IC is given in Section III. In Section IV, we show the ability of the joint diagonalization technique to decode the desired signal. The simulation results are shown in Section V. Finally, Section VI concludes the paper.

Notations: Boldface upper case letters and boldface lower case letters denote matrices and vectors, respectively. For the transpose, transpose conjugate and conjugate matrix we use $(.)^{t},(.)^{H}$ and $(.)^{*}$, respectively. |.| and $\|. \mid\|$ denote the modulus of a complex and the norm of a vector, respectively. $\mathbb{E}$ is the statistical expectation operator. 


\section{SyStem MOdEL}

We consider a $K$-user quasi static IC with $K$ transmitreceive pairs with $N$ antennas at each side of the link. A given transmitter intends to have its signal decoded by a single dedicated receiver. The received signal at the $k^{\text {th }}$ receiver node and at instant $l$ is given by

$\boldsymbol{y}_{k}(l)=\boldsymbol{H}_{k k} \boldsymbol{V}_{k} \boldsymbol{s}_{k}(l)+\sum_{j \neq k} \boldsymbol{H}_{k j} \boldsymbol{V}_{j} \boldsymbol{s}_{j}(l)+\boldsymbol{z}_{k}(l), l=$

where $L$ represents the frame length, $\boldsymbol{H}_{k j} \in \mathbb{C}^{N \times N}$ is the fading channel matrix between the $j^{\text {th }}$ transmitter and the $k^{t h}$ receiver, $\boldsymbol{V}_{j} \in \mathbb{C}^{N \times d}$ is the precoding matrix at the $j^{\text {th }}$ transmitter, and $\boldsymbol{z}_{k}(l) \in \mathbb{C}^{N \times 1}$ is the circular symmetric complex Gaussian noise vector at the $k^{\text {th }}$ receiver, with i.i.d. components; i.e. $\boldsymbol{z}_{k} \sim \mathcal{N}_{c}\left(0, \boldsymbol{I}_{N}\right) .\left\{\boldsymbol{s}_{j}(l) \in \mathcal{M}^{d \times 1} \mid l=\right.$ $0, \cdots, L-1\}$ represents the $d$ streams from the $j^{\text {th }}$ transmitter during an $L$-symbol duration interval. The symbols of $s_{j}$ are supposed i.i.d. from a finite constellation $\mathcal{M}$. The decoding is over one frame, in which the channel is supposed unchanged.

The $K$ precoders are jointly designed to satisfy the IA conditions, which can be achieved using different solutions [1], [5]. At the receiver side, the intended signal can be recovered by projecting the received signal on the interference null space. The $l^{\text {th }}$ decoded signal vector is given by

$$
\tilde{\boldsymbol{y}}_{k}(l)=\boldsymbol{U}_{k} \boldsymbol{H}_{k k} \boldsymbol{V}_{k} \boldsymbol{s}_{k}(l)+\sum_{j \neq k} \boldsymbol{U}_{k} \boldsymbol{H}_{k j} \boldsymbol{V}_{j} \boldsymbol{s}_{j}(l)+\boldsymbol{U}_{k} \boldsymbol{z}_{k}(l),
$$

where $\boldsymbol{U}_{k}$ denotes the decoding matrix that we aim to estimate. To this end, we exploit the statistical independence of the transmitted streams, and we seek at each receiver for the decoding matrix that maximizes the statistical independence between the components of the mixed received signal. Then, we introduce a few training symbols in order to remove the scaling and the permutation ambiguities.

\section{FROM THE FADING INTERFERENCE CHANNEL TO A BSS MODEL}

This section is dedicated to the definition of a linear BSS model from the MIMO IC model, assuming an IA scheme at the transmission side. Such model is required for the application of linear BSS techniques. Before going into details, let us recall the following lemma from linear algebra

Lemma 1: Let $\boldsymbol{A}_{1} \in \mathbb{C}^{N \times n}$ and $\boldsymbol{A}_{2} \in \mathbb{C}^{N \times n}(N>n)$, where $\operatorname{rank}\left(\boldsymbol{A}_{1}\right)=n$ and $\operatorname{rank}\left(\boldsymbol{A}_{2}\right)=m,(m \leq n)$ and $\operatorname{span}\left(\boldsymbol{A}_{2}\right) \subset \operatorname{span}\left(\boldsymbol{A}_{1}\right)$. Then, for every $\boldsymbol{s}_{2} \in \mathbb{C}^{n \times \overline{1}}, \exists \boldsymbol{s}_{1} \in$ $\mathbb{C}^{n \times 1}$ such that $\boldsymbol{A}_{1} \boldsymbol{s}_{1}=\boldsymbol{A}_{2} \boldsymbol{s}_{2}{ }^{1}$.

In the adopted transmission model, the received signal given in (1) can be expressed as

$$
\boldsymbol{y}_{k}(l)=\underbrace{\overline{\boldsymbol{H}}_{k}^{k} \boldsymbol{s}_{k}(l)}_{\text {Desired signal }}+\underbrace{\sum_{j \neq k} \overline{\boldsymbol{H}}_{j}^{k} \boldsymbol{s}_{j}(l)}_{\text {Interference signal }}+\boldsymbol{z}_{k}(l),
$$

where $\overline{\boldsymbol{H}}_{j}^{k}=\boldsymbol{H}_{k j} \boldsymbol{V}_{j}$. Assuming the IA conditions are satisfied, and let $\overline{\boldsymbol{H}}_{I}^{k} \in \mathbb{C}^{N \times(N-d)}$ denote the matrix spanning

\footnotetext{
${ }^{1}$ This is also valid for the case when $\boldsymbol{s}_{1}$ and $\boldsymbol{s}_{2} \in \mathbb{C}^{N \times L}$
}

the $(N-d)$-dimensional interference subspaces at receiver $k$; i.e. $\overline{\boldsymbol{H}}_{j}^{k}$ for $j \in\{1, \cdots, K\}, j \neq k$ are all spanned by $\overline{\boldsymbol{H}}_{I}^{k}$. Thus, using the lemma above, the interference subspaces can be expressed in terms of $\overline{\boldsymbol{H}}_{I}^{k}$ as

$$
\overline{\boldsymbol{H}}_{j}^{k} \boldsymbol{s}_{j}=\overline{\boldsymbol{H}}_{I}^{k} \boldsymbol{s}_{j I}(l), \quad \boldsymbol{s}_{j I}(l)=\left(\overline{\boldsymbol{H}}_{I}^{k^{H}} \overline{\boldsymbol{H}}_{I}^{k}\right)^{-1} \overline{\boldsymbol{H}}_{I}^{k^{H}} \overline{\boldsymbol{H}}_{j}^{k} \boldsymbol{s}_{j}(l) .
$$

(4) into (3) yields for $l=0, \cdots, L-1$

$$
\begin{aligned}
\boldsymbol{y}_{k}(l) & =\overline{\boldsymbol{H}}_{k}^{k} \boldsymbol{s}_{k}(l)+\overline{\boldsymbol{H}}_{I}^{k} \overline{\boldsymbol{s}}_{k}(l)+\boldsymbol{z}_{k}(l) \\
& =\left[\begin{array}{ll}
\overline{\boldsymbol{H}}_{k}^{k} & \overline{\boldsymbol{H}}_{I}^{k}
\end{array}\right]\left[\begin{array}{ll}
\boldsymbol{s}_{k}^{t}(l) & \overline{\boldsymbol{s}}_{k}^{t}(l)
\end{array}\right]^{t}+\boldsymbol{z}_{k}(l) \\
& =\boldsymbol{B}_{k} \tilde{\boldsymbol{s}}_{k}(l)+\boldsymbol{z}_{k}(l),
\end{aligned}
$$

where $\overline{\boldsymbol{s}}_{k}=\left(\boldsymbol{s}_{1 I}+\cdots+\boldsymbol{s}_{(k-1) I}+\boldsymbol{s}_{(k+1) I}+\cdots+\boldsymbol{s}_{K I}\right)$, $\boldsymbol{B}_{k} \in \mathbb{C}^{N \times N}$ is a full rank matrix that spans the union of the desired and the interference subspaces, and $\tilde{\boldsymbol{s}}_{k}(l)$ is the $N \times 1$ source vector consisting of $d$ desired streams and $(N-d)$ interference streams. (5) gives the formulation of a linear determined BSS problem where an $N$-length source vector $\tilde{\boldsymbol{s}}_{k}$ is mixed by an unknown mixing matrix $\boldsymbol{B}_{k}$ to produce $N$ mixture signals $\boldsymbol{y}_{k}$ observed from $N$ sensors. The BSS aims at separating the original streams from an observed sensor array without knowing the transmission parameters.

\section{EXTRACTION OF THE DESIRED SIGNAL USING BSS}

\section{A. Desired signal Extraction}

The BSS standard model is defined as

$$
\boldsymbol{y}(l)=\boldsymbol{A s}(l), \quad l=0, \cdots, L-1
$$

where $s(l) \in \mathbb{C}^{N \times 1}$ is the vector of $N$ statistically independent latent variables, $\boldsymbol{y}(l) \in \mathbb{C}^{N \times 1}$ is the observation vector, and $\boldsymbol{A} \in \mathbb{C}^{N \times N}$ is a full rank unknown mixing matrix. The BSS technique seeks for the demixing matrix $\boldsymbol{U}$ that maximizes the statistical independence between the estimated components $\hat{\boldsymbol{s}}(l)=\boldsymbol{U} \boldsymbol{A} \boldsymbol{s}(l) \forall l$. For the sake of the simplicity, the time index will be ignored in the remaining of this section.

It is shown in [7] that, under mild assumptions, the estimated variables $\hat{s}$ are similar to the original sources $s$ up to a permutation and scaling by a constant, i.e.

$$
\boldsymbol{U} \boldsymbol{A}=\boldsymbol{P} \boldsymbol{\Lambda},
$$

where $\boldsymbol{P}$ is a permutation matrix and $\boldsymbol{\Lambda}$ is a diagonal matrix.

The previous section shows that the MIMO IC model in (5) is similar to the BSS model except that some mutual dependencies exist between some components of $\tilde{\boldsymbol{s}}_{k}=\left[\begin{array}{ll}\boldsymbol{s}_{k}^{t} & \overline{\boldsymbol{s}}_{k}^{t}\end{array}\right]^{t}$. The first $d$ components of $s_{k}$ are mutually independent and represent the desired streams. The $(N-d)$-components $\bar{s}_{k}$ are mutually dependent and represent the interference part. This situation of dependent sources has been considered in certain recent studies, e.g. [10], [11]. Next, we show how to only extract the desired signal using the method JADE.

\section{B. Joint Approximate diagonalization of Eigenmatrices}

JADE is a well known statistical technique for solving linear determined BSS problem. It is based on the fact that the fourth order cross-cumulant of independent variables are zeros. Thus, demixing a received mixed signal as in (6) involves looking 
for the decoding matrix that makes the fourth order crosscumulant null [13]. We summarize the steps of the algorithm JADE as follows (for further details, the reader can refer to [9])

1) Step 1: Compute the whitening matrix $\boldsymbol{W}$ as the inverse square root of the sample covariance matrix of the received data. As shown in [9], $\boldsymbol{W}$ transforms $\boldsymbol{A}$ into a unitary matrix $\boldsymbol{F}=\boldsymbol{W} \boldsymbol{A}$.

2) Step 2: Form the sample 4-th order tensor ${ }^{2} Q_{z}$ of the whitened data $\boldsymbol{z}=\boldsymbol{W} \boldsymbol{y}$

3) Step 3: Compute the $N$ most significant eigenpairs of $\boldsymbol{Q}_{z}:\left\{\lambda_{n}, \boldsymbol{M}_{n} \mid n=1, \cdots, N\right\}$

4) Step 4: Perform the approximate joint diagonalization of matrices $\left\{\lambda_{n} \boldsymbol{M}_{n} \mid n=1, \cdots, N\right\}$ by a unitary matrix $U$

5) Step 5: An estimate of the source vector is $\hat{\boldsymbol{s}}=\boldsymbol{U} \boldsymbol{z}$

JADE has been proved able to separate the original streams when all streams are statistically independent. Let us now demonstrate that even in the presence of some mutually dependent components, as in (5), JADE is able to separate the mutual independent streams. For our considered problem, the $(N-d)$ interference sources are dependent in which case we can easily show ${ }^{3}$ that the set of matrices $\left\{\lambda_{n} \boldsymbol{M}_{n} \mid n=1, \cdots, N\right\}$ are not anymore jointly diagonalizable but are jointly blockdiagonalizable. In other words, for $n=1, \cdots, N$, we have the following joint matrix structure:

$$
\lambda_{n} \boldsymbol{M}_{n}=\boldsymbol{F}\left[\begin{array}{cc}
\boldsymbol{M}_{n, 1} & \mathbf{0} \\
\mathbf{0} & \boldsymbol{M}_{n, 2}
\end{array}\right] \boldsymbol{F}^{H}
$$

where $\boldsymbol{M}_{n, 1}$ are $d \times d$ diagonal matrices, $\boldsymbol{M}_{n, 2}$ are $(N-$ d) $\times(N-d)$ unstructured matrices and $\boldsymbol{F}=\boldsymbol{W} \boldsymbol{A}$. In [14], it is shown that the joint diagonalization algorithm used in the standard BSS method JADE can be used as well for the joint block diagonalization of a set of matrices. Consequently, the final transformation given by the whitening matrix and unitary transform $U$ leads to:

$$
\boldsymbol{U} \boldsymbol{W} \boldsymbol{A}=\left[\begin{array}{cc}
\boldsymbol{D}_{1} & \mathbf{0} \\
\mathbf{0} & \boldsymbol{D}_{2}
\end{array}\right]
$$

where $\boldsymbol{D}_{1}$ is a $d \times d$ diagonal matrix and $\boldsymbol{D}_{2}$ a $(N-d) \times(N-d)$ given matrix. Hence, the first $d$ entries of $\hat{\boldsymbol{s}}=\boldsymbol{U} \boldsymbol{z}$ represent the desired source signals while its remaining $N-d$ entries represent linear mixtures of the (non-desired) interference signals.

Another BSS technique is the iterative FastICA. This technique is characterized by its low computational complexity and fast convergence. It also performs close to the JADE in terms of robustness [8] (see section V).

\section{Semi-Blind separation}

The ambiguities on the scale and permutation of the estimated streams are solved thanks to a few training symbols inserted within each data frame. We denote $\boldsymbol{x}_{j} \in \mathbb{C}^{1 \times N_{s}}$ the

\footnotetext{
${ }^{2}$ The entries of $\boldsymbol{Q}_{z}$ are given by $\boldsymbol{Q}_{z}(i, j, k, l)=\operatorname{cum}\left(z_{i}, z_{j}^{*}, z_{k}, z_{l}^{*}\right)$ where cum refers to the fourth order cumulant and $z_{i}^{*}$ is the complex comjugate of the $i-t h$ entry of $\boldsymbol{z}$.

${ }^{3}$ By using the structure of $\boldsymbol{Q}_{z}$ in [9], one can directly obtain the matrix structure in (8). This derivation is omitted due to space limitation.
}

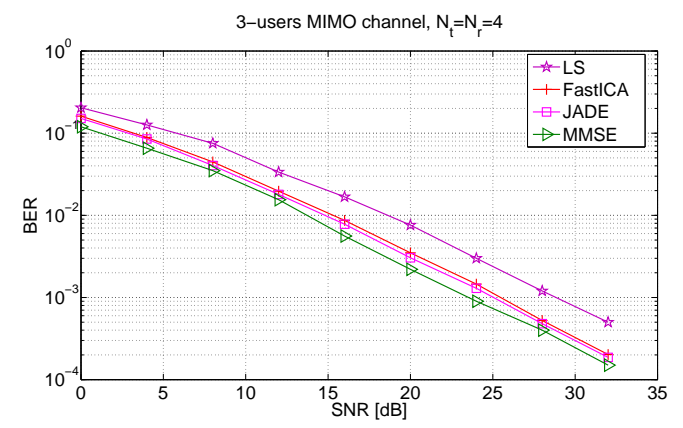

Fig. 1. BER performance comparison using $N_{s}=8$ training symbols

- FastICA: decoding scheme that uses FastICA as BSS technique.

- LS: the MMSE-based decoder with LS-CSI estimation.

- MMSE: the MMSE-based decoder with perfect CSI.

$j^{\text {th }}$ training sequence for $j \in\{1, \cdots, d\}$, and $\hat{\boldsymbol{s}}_{i}$ is the $i^{\text {th }}$ estimated independent stream with $i \in\{1, \cdots, d\}$. We define the Normalized Minimum Mean Squared Error (NMSE) as

$$
\operatorname{NMSE}\left(\hat{\boldsymbol{s}}_{i}, \boldsymbol{x}_{j}\right)=\log _{10}\left[1-\frac{\left|\hat{\boldsymbol{s}}_{i} \boldsymbol{x}_{j}^{H}\right|^{2}}{\left\|\hat{\boldsymbol{s}}_{i}|| \mid \boldsymbol{x}_{j}\right\|}\right] .
$$

The ambiguity on the permutation order is solved by minimizing the NMSE according to the training sequence

$$
\underset{i, j \in\{1, \cdots, d\}}{\operatorname{argmin}} \operatorname{NMSE}\left(\hat{\boldsymbol{s}}_{i}, \boldsymbol{x}_{j}\right),
$$

Next, the scale ambiguity can be solved by seeking for the complex variable $\alpha$ that minimizes the MMSE between the estimated variables and the training sequence

$$
\text { MMSE }=\mathbb{E}\left[\left|\alpha \hat{\boldsymbol{s}}_{j}-\boldsymbol{x}_{j}\right|^{2}\right] .
$$

The $1 \times N_{s}$ vector $\hat{\boldsymbol{s}}_{i}$ used in (9), (10), and (11) is formed by the first $N_{s}$ estimated symbols of the stream $s_{k}$.

Remarks: 1) The proposed technique requires the receiver to wait for all samples within one frame. Therefore, the authors in [15] have proposed an adaptative semi-blind high order separation technique. This technique can be adapted to our case since we have showed that the dependency between interference streams does not affect the desired source extraction. 2) As shown by our simulation results, the semi-blind approach results in a slight performance loss as compared to the standard (data-aided) MMSE. However, it is shown in [12], that in such cases one can compensate for this performance loss using a decision-directed MMSE detection in a two step approach, the first step being the semi-blind approach proposed previously (see [12] for further details).

\section{Simulation Results}

In this section, we evaluate the Bit Error Rate (BER) of the JADE and FastICA in a 3 -user $4 \times 4$ MIMO IC with an IA design. The IA scheme is achieved using the distributed iterative solution proposed in [5]. Each user sends $d=2$ data streams. The symbols are QPSK modulated. The channel is supposed flat fading Rayleigh distributed, and remains constant over one frame with length $L=2000$ symbols. 


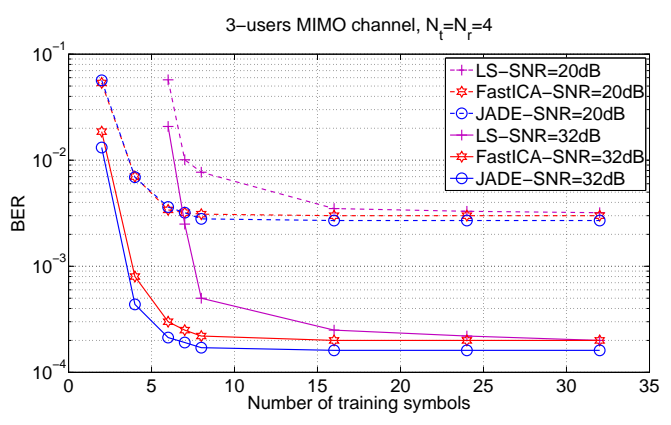

Fig. 2. The influence of the training sequence length on the BER performance

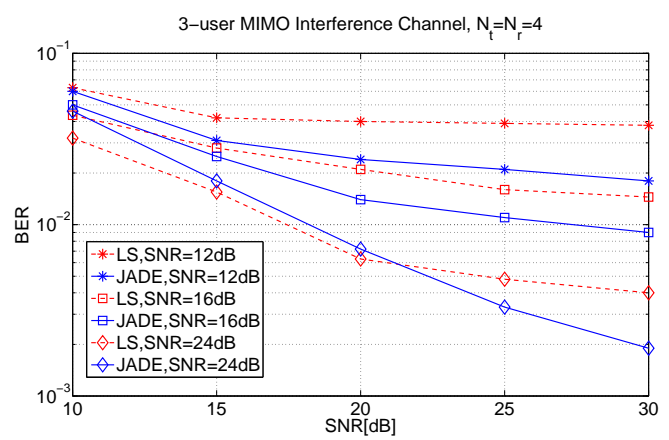

Fig. 3. Effect of the IA imperfection on the BER performance $N_{s}=8$

Fig. 1 depicts the BER performance of the MMSE-based decoder with full CSI, the BSS-based decoders (JADE and FastICA), and the MMSE-based decoder with LS-CSI estimation. We use $N_{s}=8$ symbols to reveal the scale and the permutation ambiguities, and to estimate the CSI using LS. As shown, JADE and FastICA have close BER performance in the entire SNR region. They also perform close to the MMSEbased with full CSI with a gap of about $1 \mathrm{~dB}-2 \mathrm{~dB}$ over the entire SNR region. On the other hand, compared to the LS, a gain between $1 \mathrm{~dB}-4 \mathrm{~dB}$ over all SNR values is obtained.

Next, Fig. 2 describes the BER behavior of the JADE and FastICA algorithm in terms of the training sequence length. The MMSE-LS requires at least $N_{s}=\sum_{j=1}^{3} d_{j}=6$ to separate the sources. The BSS techniques can separate the independent sources without any training symbols. However, to solve the scaling and the permutation problem, only $N_{s}=1$ symbol is necessary. At $20 \mathrm{~dB}$, the BER of both techniques improves with $N_{s}$ to reach $3.10^{-3}$ for $N_{s}=8$, and remains roughly unchanged when $N_{s}$ increases. Comparing to the LS, an important BER gain is obtained for $N_{s}<16$. This gain decreases when $N_{s}$ increases and becomes negligible at $N_{s}=32$. This BER comparison is similar for $\mathrm{SNR}=32 \mathrm{~dB}$.

Next, in order to perform the IA design, channel matrices have to be estimated. Therefore, we assume a TDD-uplink transmission scheme where the receiver sends to the transmitter an $L_{s}$-length reference signal in a reciprocal channel for CSI estimation. This strategy is employed in the 3GPP-LTE network [16]. The estimated channel matrices are modeled as [4]

$$
\tilde{\boldsymbol{H}}_{k j}=\boldsymbol{H}_{k j}+\boldsymbol{E}_{k j} ; \forall k, j
$$

where $\boldsymbol{E}_{k j}$ is the channel estimation error $\forall k, j$. The coefficients of $\boldsymbol{E}_{k j}$ are symmetric complex Gaussian distributed with zero mean and $\sigma_{e}^{2}$ variance. In Fig. 3, the BER performance of both LS and JADE methods is illustrated in presence of a channel estimation error. It can be observed that in the region where $\sigma_{h}^{2} / \sigma_{e}^{2}<10 \mathrm{~dB}$, both of the decoders results in a degraded BER. However, our proposed decoder outperforms the LS: i) beyond $\sigma_{h}^{2} / \sigma_{e}^{2}=10 \mathrm{~dB}$ when $\mathrm{SNR}=12 \mathrm{~dB}$, ii) beyond $\sigma_{h}^{2} / \sigma_{e}^{2}=14 \mathrm{~dB}$ when $\mathrm{SNR}=16 \mathrm{~dB}$, and iii) beyond $\sigma_{h}^{2} / \sigma_{e}^{2}=21 \mathrm{~dB}$ when $\mathrm{SNR}=20 \mathrm{~dB}$.

\section{CONCLUSION}

In this paper, we have adressed the problem of detection in a MIMO IC system using IA scheme at the transmitters. The problem has been formulated as a semi-blind source separation problem, and the ability of the joint diagonalization technique (JADE) has been shown to extract the desired streams. Training sequence has been introduced to solve the permutation and scaling ambiguities. The proposed scheme performs close to full-CSI MIMO IC-IA schemes. We have also showed by simulations that it outperforms the traditional MMSE using LS for CSI estimation method with a same training sequence length.

\section{REFERENCES}

[1] V. R. Cadambe and S. A. Jafar, "Interference Alignment and Degrees of Freedom of the K-user Interference Channel," IEEE Transactions on Information Theory, vol. 54, no. 8, Aug. 2008.

[2] M. Maddah-Ali, A. S. Motahari, and A. K. Khandani, "Communication over MIMO X Channels: Interference Alignment, Decomposition, and Performance Analysis," IEEE Transactions on Information Theory, vol. 54, pp. 3457-3470, Aug. 2008.

[3] C. Yetis, T. Gou, S. A. Jafar, and A. H. Kayran, "On Feasibility of Interference Alignment in MIMO Interference Networks," IEEE Transactions on Signal Processing, vol. 8, pp. 4771-4782, Sept. 2010.

[4] R. Tresch, and M. Guillaud, "Cellular Interference Alignment with Imperfect Channel Knowledge," in Proc. IEEE ICC Workshops, 2009.

[5] K. Gomadam, V. Cadambe, and S. Jafar, "Approaching the Capacity of Wireless Networks through Distributed Interference Alignment," in in Proc. of IEEE Globecom, 2008.

[6] H. Bolcskei, and J. Thukral, "Interference alignment with limited feedback," in in Proc. of IEEE ISIT, 2009.

[7] P. Comon, "Independent Component Analysis, A New Concept?" Signal Processing, pp. 287-314, 1994.

[8] E. Bingham and A. Hyvarinen, "ICA of Complex Valued Signals: a Fast and Robust Deflationary Algorithm," International Journal Neural Systems, vol. 10, no. 1, Feb. 2000.

[9] J-F. Cardoso and A. Souloumiac, "Blind Beamforming for Non-Gaussian Signals", IEE-Proceedings F, vol. 140, no. 6, pp. 362-370, Dec. 1993.

[10] M. Castella and P. Comon, "Blind Separation of Instantaneous Mixtures of Dependent Sources", in Proc. of ICA Workshop, 2007.

[11] Cesar F. Caiafa, "On the conditions for valid objective functions in blind separation of independent and dependent sources", EURASIP Journal on Advances in Signal Processing, Dec. 2012.

[12] I. Kacha, K. Abed-Meraim and A. Belouchrani, "Fast adaptive blind MMSE equalizer for multichannel FIR systems", EURASIP Journal on Applied Signal Processing, vol. 2006, 2006.

[13] J. F. Cardoso, "High-order contrasts for independent component analysis," Neural Computation, vol. 11, no. 1, pp. 157-192, 1999.

[14] H. Bousbia-Salah, "Blind separation of source signals from their convolutive mixtures: Bloc Joint Diagonalization technique based on second order statistics", PhD thesis at ENP, , Algeria, May 2006.

[15] D. Zhiguo, T. Ratnarajah, and C. Cowan, "HOS-Based Semi-Blind Spatial Equalization for MIMO Rayleigh Fading Channels," IEEE Transactions on Signal Processing, vol. 56, pp. 248-255, 2008.

[16] ETSI 3GPP, "LTE; Evolved Universal Terrestrial Radio Access (EUTRA); and Evolved Universal Terrestrial Radio Access Network (EUTRAN); overall description," 3GPP TS 36.300 version 9.4.0 Release 9.0. 